\title{
Revisión de celulitis periorbitaria y orbitaria. Experiencia de quince años
}

\author{
E. La Orden Izquierdo a M. Ruiz Jiménez ${ }^{\mathrm{b}}$, JA. Blázquez Fernández \\ M. Prados Álvarez ${ }^{\mathrm{a}}$, MD. Martín Pelegrina ${ }^{\mathrm{a}}$, JT. Ramos Amador ${ }^{\mathrm{b}}$ \\ "MIR Pediatría. \\ 'Pediatra. Servicio de Pediatría, Hospital Universitario de Getafe. Getafe, Madrid. España.
}

\author{
Rev Pediatr Aten Primaria. 2009; 1 1:597-606 \\ Enrique La Orden Izquierdo, e_o_izquierdo@yahoo.es
}

\section{Resumen}

Introducción: las infecciones oculares son frecuentes en la edad pediátrica. La distinción entre periorbitaria y orbitaria es fundamental por el diferente pronóstico. Su diagnóstico y tratamiento precoces son importantes porque pueden producir complicaciones graves.

Objetivos: revisar la epidemiología, las manifestaciones clínicas y el diagnóstico, la microbiología, las complicaciones y el tratamiento de la celulitis periorbitaria y orbitaria en niños menores de 15 años ingresados por esta patología.

Material y métodos: estudio retrospectivo de pacientes menores de 15 años ingresados por celulitis periorbitaria y orbitaria desde abril de 1992 hasta diciembre de 2006 en el Hospital Universitario de Getafe (Madrid). Los criterios clínicos que se siguieron para el diagnóstico de la celulitis periorbitaria fueron la presencia de signos inflamatorios en tejidos blandos periorbitarios y/u oculares, incluyendo al menos hinchazón palpebral e inyección conjuntival. Signos como la disminución de la agudeza visual, alteración de la motilidad ocular, quemosis, proptosis, alteraciones del fondo de ojo y afectación sistémica orientaban a celulitis orbitaria. En casos dudosos, el diagnóstico de localización se estableció mediante la tomografía computarizada.

Resultados: se incluyó a 66 pacientes, 36 de los cuales (55\%) eran varones. La mediana de edad fue de 38 meses (rango: 3 meses a 14 años). Las patologías asociadas fueron: conjuntivitis (26\%), infección de la vía respiratoria superior (42\%), patología dental (6\%), puerta de entrada cutánea (12\%) y otros (14\%). En 19 de los 27 pacientes en los que se realizaron técnicas de imagen se objetivó sinusitis asociada. Los patógenos considerados más frecuentes fueron Staphylococcus aureus y Streptococcus pneumoniae, y en menor cuantía Haemophilus influenzae. Todos los pacientes recibieron tratamiento antibiótico intravenoso: un $36 \%$ en monoterapia (cefuroxima, amoxicilina-clavulánico) y un $64 \%$ en politerapia, asociando corticoides sistémicos en un $24 \%$ y tratamiento quirúrgico local en un 7,5\%. Todos los pacientes mejoraron y no tuvieron secuelas posteriores.

Los autores declaran no presentar conflictos de intereses en relación con la preparación y publicación de este artículo. Corresponde parcialmente al contenido de un póster presentado en el 56. ${ }^{\circ}$ Congreso de la AEP en Barcelona, 2007. 
Conclusiones: la celulitis periorbitaria y orbitaria es un proceso habitual en pediatría y potencialmente grave. Suele ocurrir en menores de 4 años, y en niños mayores es frecuente su asociación con sinusitis. La rentabilidad de los hemocultivos es baja frente a la de los exudados oculares. Suelen tener una evolución favorable con resolución del cuadro en las primeras 48 horas, salvo complicación, si el diagnóstico y el tratamiento son precoces.

Palabras claves: Celulitis periorbitaria, Microbiología, Tratamiento.

\section{Abstract}

Introduction: eye infections are frequent in children. The distinction between periorbital and orbital is essential because of its different prognosis. Early detection and treatment are important because they can produce serious complications.

Objective: to check the epidemiology, clinical symptoms and prognosis, microbiology, complications and periorbital/orbital cellulitis treatment in children younger than fifteen admitted into hospital due to this pathology.

Patients and methods: retrospective study of patients under 15 years hospitalized because of periorbital cellulitis from April of 1992 to December of 2006 in Getafe University Hospital. The clinical criterions followed to diagnose periorbital cellulitis were the presence of inflammatory signs in eye or periorbital soft tissues; including at least palpebral swelling and conjunctival injection. Signs like the disminution of visual acutennes, the alteration of the eye motility, chemosis, proptosis, alterations of the fundus examination and systemic symptoms orientated to orbital cellulitis. In doubtful cases, the location diagnosis was established by TAC.

Results: sixty six patients were included and thirty six (55\%) were males. The median of the age was 38 months (rank: 3 months-14 years). The associated pathology was: conjunctivitis $26 \%$, upper respiratory tract infections $42 \%$, dental pathology $6 \%$, local cutaneous trauma $12 \%$ and others $14 \%$. Associated sinusitis was present in 19 of the 27 patients who underwent image technique. The more frequent pathogens considered were Staphylococcus aureus and Streptococcus pneumoniae, and with less frequency Haemophilus influenzae. All the patients received an intravenous antibiotic treatment: $36 \%$ with a single antibiotic (cefuroxime or amoxicillin-clavulanic acid) and 64\% multiple antibiotic therapy, associating systemic corticosteroids in the $24 \%$ and local surgical treatment in the $7.5 \%$. All the patients improved and they had not sequelae.

Conclusions: the periorbital/orbital cellulitis is a frequent and potentially serious process in pediatrics. It usually appears in children under four, and its association with sinusitis is frequent in older children. The efficiency of blood cultures is low compared to eye exudates. They usually have a favourable evolution with the resolution of the disease in the first 48 hours, except complication, with early diagnosis and treatment.

Key words: Periorbital cellulitis, Microbiology, Treatment.

\section{Introducción}

Las infecciones oculares son frecuentes en la edad pediátrica. La distinción entre periorbitaria y orbitaria es fundamental porque el abordaje terapéutico y el pronóstico son diferentes. Su diagnóstico y tratamiento precoces son importantes porque pueden producir complicaciones graves. En la práctica clínica es importante que el pediatra 
mantenga una colaboración activa con radiólogos, otorrinos y oftalmólogos. Existen pocas series descritas en población infantil en nuestro país; de ahí la relevancia de su conocimiento. El objetivo de este trabajo es la revisión de la epidemiología, las manifestaciones clínicas, las complicaciones y el tratamiento de la celulitis periorbitaria y orbitaria en este grupo de población.

\section{Pacientes y métodos}

Estudio retrospectivo de pacientes menores de 15 años ingresados por celulitis periorbitaria y orbitaria desde abril de 1992 hasta diciembre de 2006 en el Hospital Universitario de Getafe (Madrid). Nuestro hospital atiende a una población menor de 15 años de 49.621 habitantes. El número de urgencias pediátricas fue de 37.424 en el año 2006.

Los criterios clínicos que se siguieron para el diagnóstico de la celulitis periorbitaria fueron la presencia de signos inflamatorios en tejidos blandos periorbitarios y/u oculares, incluyendo al menos hinchazón palpebral e inyección conjuntival.

Se recogieron datos clínicos procedentes de las historias clínicas de los pacientes con diagnóstico de celulitis periorbitaria/orbitaria al ingreso. La presencia de signos como la disminución de la agudeza visual, alteración de la motilidad ocular, quemosis, proptosis, alteraciones del fondo de ojo y afectación sistémica obligaban a descartar la afectación orbitaria. En casos dudosos, el diagnóstico de localización se estableció mediante la tomografía computarizada (TC).

Las variables analizadas fueron: sexo, edad, peso del paciente, mes de aparición, patología asociada, días de evolución previos al ingreso, días que el paciente permanece sintomático durante el ingreso, tratamientos recibidos previos al ingreso, presencia de fiebre, localización (izquierda, derecha o bilateral), presencia de edema palpebral y su localización (superior, inferior o ambos), asociación con sinusitis, cefalea, hiperemia conjuntival y exudación ocular, dolor ocular, quemosis, proptosis, visión borrosa, nistagmo, alteración de la motilidad ocular extrínseca e intrínseca, alteraciones en el fondo de ojo y complicaciones evolutivas; se usaron una base de datos Access $^{\circledast} 2007$ y una hoja de cálculo Excel $^{\oplus}$ 2007. Dentro del apartado de diagnóstico se analizaron las técnicas de imagen realizadas, las edades en que se aplicaron y los hallazgos obtenidos, los parámetros analíticos -valores de proteína $C$ reactiva $(P C R)$, leucocitosis y fórmula leucocitaria-, las 
muestras microbiológicas y el resultado de éstas. Con respecto al tratamiento se recogieron el régimen de tratamiento intravenoso (IV) administrado, su duración, el uso de tratamiento antibiótico tópico asociado, la necesidad de tratamiento antibiótico oral posterior y su duración, la administración de corticoides sistémicos y la aplicación de tratamiento quirúrgico local, así como la duración de la estancia hospitalaria.

\section{Resultados}

De un total de 66 pacientes revisados, $36(55 \%)$ eran varones. La mediana de edad fue de 38 meses, con un rango de edad de 3 meses a 14 años. Las patologías asociadas fueron: conjuntivitis $(26 \%)$, infección de la vía respiratoria superior $(42 \%)$, patología dental $(6 \%)$, puerta cutánea -picaduras, heridas, etc.- $(12 \%)$ y otros $(14 \%)$-dacriocistitis, otitis media aguda, fiebre faringoconjuntival, faringoamigdalitis o ninguna causa atribuible-. La localización fue izquierda en un $51 \%$ de los casos, derecha en un $44 \%$ y bilateral en un $5 \%$. El $20 \%$ de los pacientes recibió tratamiento antibiótico oral previo al ingreso. El $74 \%$ de los casos se identificó en las primeras 24 horas de evolución. Los pacientes permanecieron sintomáticos

Tabla I. Características de los 66 casos de celulitis periorbitaria en el Hospital Universitario de Getafe desde abril de 1992 hasta diciembre de 2006

\begin{tabular}{|c|c|}
\hline Sexo & $\begin{array}{l}\text { Varones: } 55 \%(n=36) \\
\text { Mujeres: } 45 \%(n=30)\end{array}$ \\
\hline Edad & Mediana de edad: 38 meses ( 3 meses-14 años) \\
\hline Patología asociada & $\begin{array}{l}\text { Conjuntivitis: } 26 \%(n=17) \\
\text { IVRS: } 42 \%(n=28) \\
\text { Patología dental: } 6 \%(n=4) \\
\text { Puerta cutánea: } 12 \%(n=8) \\
\text { Otros }^{*}: 14 \%(n=9)\end{array}$ \\
\hline Localización & $\begin{array}{l}\text { Izquierda: } 51 \%(n=34) \\
\text { Derecha: } 44 \%(n=29) \\
\text { Bilateral: } 5 \%(n=3)\end{array}$ \\
\hline Tratamiento antibiótico previo & $\begin{array}{l}\text { Sí: } 20 \%(n=13) \\
\text { No: } 53 \%(n=53)\end{array}$ \\
\hline Días de evolución previos al ingreso & $\begin{array}{l}\text { Menos de } 24 \text { horas: } 74 \%(n=49) \\
\text { Más de } 24 \text { horas: } 26 \%(n=17)\end{array}$ \\
\hline Días sintomáticos desde el ingreso & Mediana: 48 horas ( $1-6$ días) \\
\hline
\end{tabular}


desde su ingreso una mediana de 48 horas (1-6 días). La estacionalidad observada en este período fue: en otoñoinvierno (de octubre a marzo), un 55\%, y en primavera-verano (de abril a septiembre), un $45 \%$. Todos estos datos se reflejan en las tablas I y II.

Los hallazgos clínicos al ingreso fueron: fiebre $(100 \%)$, edema palpebral (100\%), hiperemia y exudación ocular $(50 \%)$ y dolor (26\%). Sólo un paciente fue diagnosticado en el ingreso de posible celulitis orbitaria. Tras aplicar una técnica de imagen (TC), el diagnóstico fue de celulitis periorbitaria (figura 1).

En cuanto a los resultados analíticos, encontramos una leucocitosis mayor de $12.500 / \mathrm{mm}^{3}$ en un $59 \%$ de los casos con una neutrofilia mayor del $65 \%$ en un $41 \%$ de los pacientes. Los valores de la PCR fueron mayores de $100 \mathrm{mg} / \mathrm{l}$ en un $13 \%$ de los casos, entre 50 y 100

Tabla II. Reparto en números absolutos de la patología asociada según la época del año

\begin{tabular}{l|c|c|c|c|c|c}
\hline & Conjuntivitis & IVRS & $\begin{array}{c}\text { Patología } \\
\text { dental }\end{array}$ & $\begin{array}{c}\text { Puerta de } \\
\text { entrada cutánea }\end{array}$ & Resto & Total \\
\hline $\begin{array}{l}\text { Primavera-verano } \\
\text { (abril-septiembre) }\end{array}$ & 7 & 9 & 3 & 4 & 7 & 30 \\
\hline $\begin{array}{l}\text { Otoño-invierno } \\
\text { (octubre-marzo) }\end{array}$ & 10 & 19 & 1 & 4 & 2 & 36 \\
\hline Total & 17 & 28 & 4 & 8 & 9 & 66 \\
\hline IVRS: catarro de las vías altas y sinusitis. & & & & & \\
\hline
\end{tabular}

Tabla III. Resultados microbiológicos en 66 pacientes con celulitis

\begin{tabular}{l|c|c|c}
\hline & \multicolumn{3}{|c}{ Tipo de muestra } \\
\cline { 2 - 4 } & Hemocultivo & Exudado ocular & Exudado herida \\
\hline Total de positivos & $53(80 \%)$ & $32(48 \%)$ & \\
\hline - Staphylococcus aureus* & $1(2 \%)$ & $25(78 \%)$ & $3(4,5 \%)$ \\
\hline - Streptococcus pneumoniae & 1 & 5 & - \\
\hline - Haemophilus influenzae & - & 5 & - \\
\hline - Streptococcus pyogenes & - & 3 & - \\
\hline - Streptococcus viridans & - & - & - \\
\hline - Staphylococcus coagulasa negativo & - & 10 & - \\
\hline - Staphylococcus auricularis & - & 9 & - \\
\hline - Streptococcus sp. & - & 1 & - \\
\hline - Enterococcus sp. & - & 1 & - \\
\hline - Bacillus sp. & - & 1 & \\
\hline * En el paciente con el hemocultivo positivo para Staphylococcus aureus no se encontró dicho \\
\hline germen en el exudado ocular realizado. & \multicolumn{3}{|c}{} \\
\hline
\end{tabular}


$\mathrm{mg} / \mathrm{l}$ en un $22,2 \%$, entre 20 y $50 \mathrm{mg} / \mathrm{l}$ en un $22,2 \%$ y menor de $20 \mathrm{mg} / \mathrm{l}$ en un $18 \%$ de los pacientes.

Se aplicó una técnica de imagen en 27 pacientes: 22 radiografías de senos paranasales, 4 TC y 1 ortopantomografía. La mediana de edad del grupo en el que se realizó la técnica de imagen fue de 60 meses (14-171 meses). Dentro de este grupo se objetivaron 19 casos de sinusitis asociada. En todos ellos se encontraba afectado el seno maxilar, mientras que los senos frontales y etmoidales sólo estaban afectados en 2 y 8 pacientes respectivamente. La mediana de edad en la que se objetivó una afectación sinusal fue de 69 meses (21-171 meses).
Se recogieron 53 hemocultivos, de los cuales sólo uno fue positivo para Staphylococcus aureus. Se obtuvieron 32 exudados oculares y se aislaron microorganismos en 25. Las bacterias consideradas patógenos más habituales fueron Staphylococcus aureus y Streptococcus pneumoniae. El Haemophilus influenzae fue aislado en 3 exudados oculares (uno de ellos de tipo B), pero en ningún hemocultivo. El resto de patógenos aislados fueron considerados como probables contaminantes. Estos resultados están resumidos en la tabla III.

Todos los pacientes recibieron tratamiento antibiótico intravenoso inicialmente: un $36 \%$ en monoterapia (cefuro-

Figura 1. Tumefacción preseptal de partes blandas que produce proptosis ocular. Se descarta afectación perióstica. Ocupación por densidad de las partes blandas de senos etmoidales y maxilares

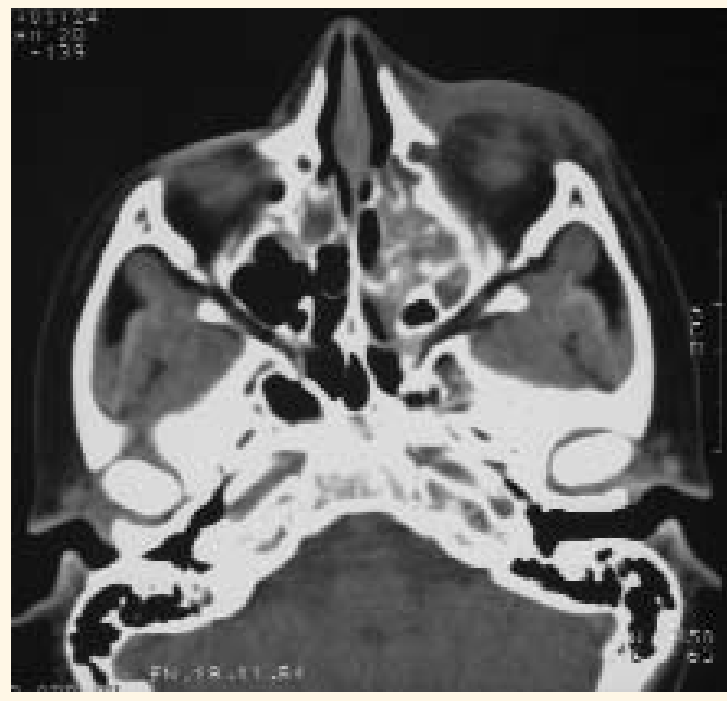


xima y, más recientemente, amoxicilinaclavulánico) y un $64 \%$ en politerapia (cloxacilina y cefotaxima y, en algunos casos, cloxacilina y cefuroxima). Se asoció el tratamiento antibiótico tópico con colirio de tobramicina o de polimixina B y neomicina en 41 pacientes (62\%); recibieron corticoides intravenosos $(1-2 \mathrm{mg} / \mathrm{kg} / \mathrm{dí}$ de metilprednisolona) 16 pacientes (24\%); y, por último, requirieron tratamiento quirúrgico local (incisión y drenaje local de herida) 5 pacientes (7,5\%). La mediana de la duración total del tratamiento antibiótico fue de 11 días (7-23 días): intravenoso, 4 días (2-11 días), y oral, 7 días (4-20 días). La estancia media hospitalaria fue de 5 días (2 a 11 días).

Todos los pacientes mejoraron y no tuvieron secuelas posteriores. En uno de ellos se confirmó una infección por el virus del herpes simple tipo 1 mediante inmunofluorescencia directa y actualmente continúa en seguimiento por queratitis en el servicio de oftalmología.

\section{Discusión}

La celulitis periorbitaria y la orbitaria realmente son dos momentos evolutivos de una misma entidad. Su diagnóstico se basa en la localización anatómica de la reacción inflamatoria. Atendiendo a dicho criterio, y según los resultados obtenidos por la TC, podemos definir cuatro estadios ${ }^{1,2}$ de este proceso: grupo 1: celulitis preseptal o preorbitaria: edema periorbitario sin afectación de las estructuras intraorbitarias; grupo 2: absceso periorbitario o subperióstico: pus entre la periórbita y la pared ósea; grupo 3: celulitis orbitaria: afectación del contenido orbitario con proptosis, disminución de la agudeza visual y diplopía; grupo 4: absceso orbitario: el pus llega a la órbita y existe riesgo de flebitis y trombosis cavernosa.

En la valoración clínica, signos como la proptosis, la quemosis conjuntival, el dolor con el movimiento ocular o la oftalmoplejía hacen sospechar una probable afectación orbitaria por compresión y empuje de dichas estructuras, y su aparición debe ser una indicación de la TC para el diagnóstico de confirmación y extensión ${ }^{1-4}$. También un gran componente inflamatorio palpebral, que impida una correcta exploración ocular, debe ser una indicación de la TC. Esta patología es relativamente frecuente y potencialmente grave en la edad pediátrica por el bajo espesor óseo que separa los tejidos periorbitarios de las estructuras orbitarias y cerebrales, lo cual facilita la propagación de procesos infecciosos a este nivel ${ }^{2,5}$.

Su forma de comenzar está en relación con cualquier proceso que produzca 
enrojecimiento y tumefacción palpebral, tales como picaduras, traumatismos, procesos alérgicos o edema inflamatorio secundario a sinusitis. En nuestra serie, la patología asociada hallada más frecuentemente fue la infección de la vía respiratoria superior (IVRS), incluyendo dentro de ella los catarros de las vías altas y las sinusitis en un $42 \%$ de los casos, seguida de patología dental (6\%), puerta cutánea -picaduras, heridas, etc.- (12\%) y otros-dacriocistitis, otitis media, fiebre faringoconjuntival, faringoamigdalitis o ninguna causa atribuible- (14\%). Estos datos son similares a los recogidos en otras series ${ }^{1,5}$.

Nuestra mediana de edad para las celulitis preseptales (38 meses) fue menor en comparación con la media obtenida por el grupo de Rodríguez Ferrán y cols. en su revisión de 107 casos, que fue de 4,3 años. Sin embargo, todas las series estudiadas coinciden en la relación directa existente entre la edad del paciente y la gravedad del proceso ${ }^{5}$, de tal forma que en menores de 5 años es más frecuente la celulitis periorbitaria mientras que en mayores de dicha edad es más habitual la celulitis orbitaria. Coincidimos en nuestra serie en la aparición de un predominio de la afectación en el sexo masculino y del lado izquierdo, al igual que sucede en otras series descri- tas, pero dicho predominio no ha sido estadísticamente significativo en ninguna de ellas ${ }^{1,4}$. También nuestro patrón estacional es similar; existe un predominio en los meses fríos relacionado probablemente con la mayor incidencia de IVRS en esta época'.

Los patógenos considerados como más frecuentes en nuestra serie fueron el Staphylococcus aureus y el Streptococcus pneumoniae. Sólo se aislaron tres Haemophilus influenzae y de ellos, uno fue del tipo $b$, de dudoso valor patógeno. Los exudados oculares muestran con frecuencia un crecimiento de posibles patógenos, si bien su papel etiológico es cuestionable. Resultados similares han sido recogidos en otras series ${ }^{1,3}$. Tanto por nuestros datos, como por los encontrados en otras series similares, señalamos la baja rentabilidad de los hemocultivos frente a la de los exudados oculares'. El resultado de nuestra bacteriología queda resumido en la tabla III.

En cuanto al tratamiento, todas las celulitis periorbitarias suelen requerir el ingreso por su potencial gravedad, si bien algunas pueden ser tratadas ambulatoriamente, con un control exhaustivo y previa información a los padres sobre la posibilidad de empeoramiento. Como tratamiento empírico inicial nosotros proponemos, por su relación patogénica 
con las sinusitis, y tras la revisión de artículos relacionados, el uso de amoxicilinaclavulánico IV para las celulitis preseptales, y la combinación de cloxacilina y cefotaxima IV para las orbitarias ${ }^{4,6}$. Es importante la toma de muestras previa a la instauración del tratamiento para adecuar éste a los resultados microbiológicos. El tratamiento quirúrgico podrá ser necesario en función del grado de extensión del proceso. En el abordaje terapéutico de la celulitis orbitaria será necesario un equipo multidisciplinar que involucre a pediatras, oftalmólogos, radiólogos y, ocasionalmente, neuropediatras.

El uso de corticoides sistémicos en esta patología sigue siendo un tema controvertido. No existe evidencia suficiente para aconsejar dicha práctica, aunque algunos estudios apuntan que podrían ser de ayuda en el manejo de las celulitis orbitarias. Hacen falta estudios controlados y aleatorizados que ayuden a dilucidar su papel terapéutico en esta patología?.
Nuestro estudio está limitado por su diseño retrospectivo, no basado en un protocolo consensuado, y con un número relativamente pequeño de casos, ninguno de los cuales tuvo diagnóstico de celulitis orbitaria, lo cual refleja la experiencia extraída de nuestra población de referencia. Como conclusiones de nuestro trabajo podemos afirmar que, en nuestro medio, la mayoría de los casos de celulitis preseptales se producen en menores de 4 años. Asimismo, queremos recalcar la frecuente asociación con la sinusitis. Por otro lado, nuestro estudio confirma la baja rentabilidad de los hemocultivos. Finalmente, las celulitis preseptales son frecuentes en la infancia y tienen una evolución favorable con resolución del cuadro en las primeras 48 horas salvo complicaciones ${ }^{8}$. Esta evolución depende principalmente del diagnóstico precoz de la extensión del proceso y de la instauración lo más pronto posible de un tratamiento antibiótico adecuado para su resolución.

\section{Bibliografía}

1. Rodríguez Ferrán $L$, Puigarnau Vallhonrat $R$, Fasheh Youssef W, Ribó Aristazábal JL, Luaces Cubells C, Pou Fernández J. Celulitis orbitaria y periorbitaria. Revisión de 107 casos. An Esp Pediatr. 2000;53:567-72.

2. Howe L, Jones NS. Guidelines for the mana- gement of periorbital cellulitis/abscess. Clin Otolaryngol. 2004;29:725-8.

3. Starkey CR, Steele RW. Medical management of orbital cellulitis. Pediatr Infect Dis J. 2001;20:1002-5.

4. Savithri N, Woods CR, Daniel BK, Givner LB, Shetty AK. Orbital Cellulitis in Children. Pediatr Infect Dis J. 2006;25(8):695-9. 
5. Gómez Campderá JA. Complicaciones de las sinusitis en la infancia. An Pediatr (Monogr). 2003;1(1):40-6.

6. González de Dios J, Ochoa Sangrador C, Álvarez Calatayud G. Manejo racional de la antibioterapia en las infecciones otorrinolaringológicas en la infancia: revisión crítica de las mejores pruebas científicas. Acta Otorrinolaringol Esp. 2006;57:66-81.
7. Yen MT, Yen KG. Effect of Corticosteroids in the Acute Management of Pediatric Orbital Cellulitis with subperiosteal abscess. Ophthal Plast Reconstr Surg. 2005;21(5):363-7.

8. Sorin A, April MM, Ward RF. Recurrent Periorbital Cellulitis: An Unusual Clinical Entity. Otolaryngol Head\&Neck Surg. 2006;134(1): 153-6. 